\title{
Cholinergic Synaptic Transmission in Adult Drosophila Kenyon Cells In Situ
}

\author{
Huaiyu Gu and Diane K. O'Dowd \\ Department of Anatomy and Neurobiology and Department of Developmental and Cell Biology, University of California at Irvine, Irvine, California 92697- \\ 1280
}

Behavioral and genetic studies in Drosophila have contributed to our understanding of molecular mechanisms that underlie the complex processes of learning and memory. Use of this model organism for exploration of the cellular mechanisms of memory formation requires the ability to monitor synaptic activity in the underlying neural networks, a challenging task in the tiny adult fly. Here, we describe an isolated whole-brain preparation in which it is possible to obtain in situ whole-cell recordings from adult Kenyon cells, key members of a neural circuit essential for olfactory associative learning in Drosophila. The presence of sodium action potential (AP)-dependent synaptic potentials and synaptic currents in $>50 \%$ of the Kenyon cells shows that these neurons are members of a spontaneously active neural circuit in the isolated brain. The majority of sodium AP-dependent synaptic transmission is blocked by curare and by $\alpha$-bungarotoxin ( $\alpha$-BTX). This demonstrates that nicotinic acetylcholine receptors (nAChRs) are responsible for most of the spontaneous excitatory drive in this circuit in the absence of normal sensory input. Furthermore, analysis of sodium AP-independent synaptic currents provides the first direct demonstration that $\alpha$-BTX-sensitive nAChRs mediate fast excitatory synaptic transmission in Kenyon cells in the adult Drosophila brain. This new preparation, in which whole-cell recordings and pharmacology can be combined with genetic approaches, will be critical in understanding the contribution of nAChR-mediated fast synaptic transmission to cellular plasticity in the neural circuits underlying olfactory associative learning.

Key words: cholinergic transmission; nAChRs; $\alpha$-bungarotoxin; mushroom bodies; whole-cell recording; Kenyon cells

\section{Introduction}

In all animals, the ability to learn and recall information arises from the capacity of experience to induce persistent alterations in the underlying neural circuitry. Changes in synaptic connectivity and/or intrinsic membrane properties of one or more elements of a circuit activated during learning represent cellular substrates of memory formation (Marder et al., 1996; Milner et al., 1998). Behavioral studies in the fruit fly Drosophila melanogaster, using an extensive library of mutant and transgenic animals, have resulted in identification of a number of genes and signal cascades that contribute to memory formation (Davis, 2004). In addition, physiological recordings in Drosophila, at the neuromuscular junction, from neurons in primary culture and in the CNS of embryos and larvae, have expanded our understanding of the molecular mechanisms regulating synaptic plasticity and neuronal excitability (Zhong and Wu, 1991; Broadie et al., 1997; Lee and O'Dowd, 2000; Renger et al., 2000; Yao and Wu, 2001; Hodges et al., 2002; Park et al., 2002; Rohrbough and Broadie, 2002; Baines, 2003; Hou et al., 2003; Rohrbough et al., 2003). However, limited electrophysiological access to neurons in cen-

Received June 9, 2005; revised Nov. 7, 2005; accepted Nov. 10, 2005.

This work was supported by National Institutes of Health Grants NS27501 and DA14960 to D.K.0. We thank Drs. J. M. Campusano, H. Su, T. Holmes, and M. A. Smith for comments on previous versions of this manuscript.

Correspondence should be addressed to Dr. Diane K. O'Dowd, Department of Anatomy and Neurobiology, 112 Irvine Hall, University of California at Irvine, Irvine, CA 92697-1280. E-mail: dkodowd@uci.edu. DOI:10.1523/JNEUROSCI.4109-05.2006

Copyright $\odot 2006$ Society for Neuroscience $\quad$ 0270-6474/06/260265-08\$15.00/0 tral circuits in the adult fly has precluded exploration of the direct cellular links that mediate the translation of changes in gene expression to memory formation.

The mushroom bodies, bilaterally symmetric regions in the insect brain, are association areas necessary for olfactory learning and memory (Heisenberg, 2003). In Drosophila, each mushroom body contains $\sim 2500$ Kenyon cells, principal neurons with small, densely packed cell bodies that are located in the dorsal protocerebrum. Electrophysiological studies in the locust and honey bee indicate that Kenyon cells receive olfactory information, the conditioned stimulus during olfactory associative conditioning, directly from cholinergic projection neurons located in the antennal lobes and indirectly via GABAergic lateral horn neurons (Oleskevich, 1999; PerezOrive et al., 2002). The neural circuits activated by the unconditioned stimulus are unknown. However, genetic and behavioral evidence from Drosophila suggests that plasticity contributing to acquisition and storage of memory occurs at synapses on Kenyon cell dendrites (Dubnau et al., 2001; McGuire et al., 2001). Given the integrative function of Kenyon cells in the adult brain, the ability to evaluate synaptic transmission and plasticity in these neurons is critical to understanding cellular mechanisms underlying olfactory associative learning.

To achieve this goal, we developed a preparation to record from identified Kenyon cells in entire brains isolated from adult flies. We show that Kenyon cells in the isolated brain are mem- 


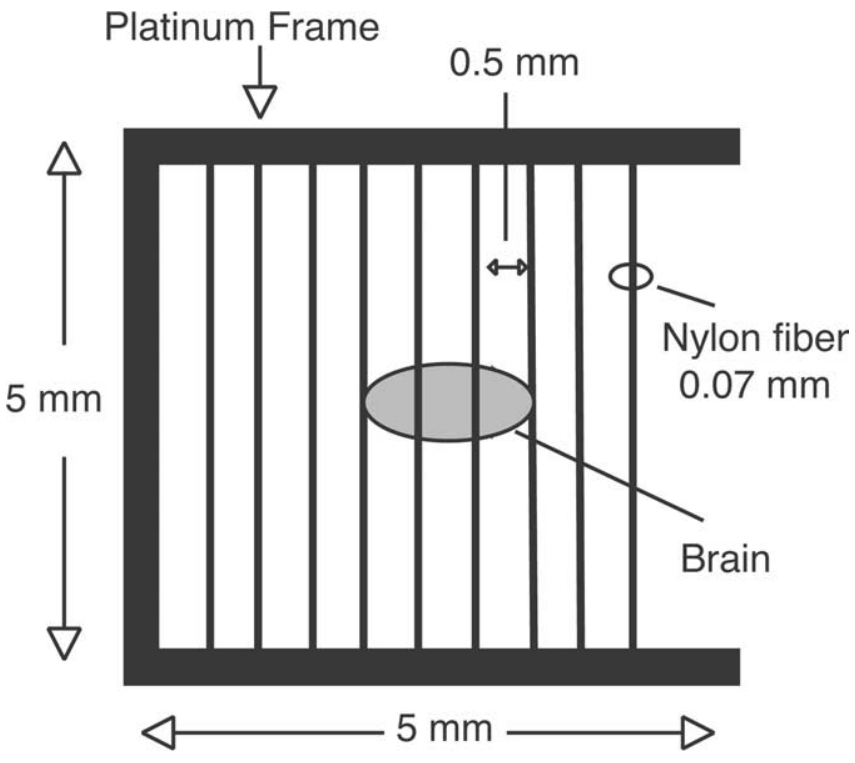

Figure 1. Holder used to stabilize isolated whole brain in the recording chamber. The U-shaped frame was fashioned, by hand, from platinum wire $(0.5 \mathrm{~mm}$ diameter; World Precision Instruments, Sarasota, FL). Nylon fibers ( $0.07 \mathrm{~mm}$ diameter) were glued to the frame, separated by $\sim 0.5 \mathrm{~mm}$, as shown. The holder was placed on top of the brain to stabilize the tissue during the recording. Brains were oriented perpendicular to cross hairs, with the fibers contacting the tissue at the borders between the central brain region and the optic lobes.

bers of a spontaneously active neural circuit in which $\alpha$-bungarotoxin ( $\alpha$-BTX)-sensitive nicotinic acetylcholine receptors (nAChRs) mediate fast excitatory synaptic transmission.

\section{Materials and Methods}

Fly stocks. Drosophila stocks were maintained on standard cornmeal medium supplemented with dry yeast. Males from the homozygous enhancer trap line OK107-GAL4 or 201Y-GAL4 were mated to females homozygous for a UAS-GFP transgene. The OK107-GAL4;UAS-GFP (OK107) animals exhibited cytoplasmic expression of green fluorescent protein (GFP) in most of the mushroom-body Kenyon cells of the adult brain (Su and O'Dowd, 2003). In the 201Y-GAL4;UAS-GFP (201Y) animals, GFP expression was limited to a subpopulation of Kenyon cells with the most prominent labeling associated with cells that project to the $\gamma$ lobe (Yang et al., 1995; Connolly et al., 1996). Wild-type flies were a Canton-S stock.

Isolated whole-brain preparation. All brains were obtained from adult female flies between 1 and $5 \mathrm{~d}$ after eclosion. The entire brain, including the optic lobes with attached ommatidia, was removed from the head in control recording saline containing the following (in $\mathrm{mm}$ ): $101 \mathrm{NaCl}, 1$ $\mathrm{CaCl}_{2}, 4 \mathrm{MgCl}_{2}, 3 \mathrm{KCl}, 5$ glucose, $1.25 \mathrm{NaH}_{2} \mathrm{PO}_{4}$, and $20.7 \mathrm{NaHCO}_{3}, \mathrm{pH}$ $7.2,250 \mathrm{mOsm}$. Each brain was incubated in recording saline containing papain $(20 \mathrm{U} / \mathrm{ml}$ activated by $1 \mathrm{~mm}$ L-cysteine; Worthington, Freehold, NJ) for 3-10 min, to soften the connective tissue sheath surrounding the brain, and washed briefly in enzyme-free oxygenated recording saline. The brain was transferred to a glass-bottom chamber for physiological recording. In the recording chamber, the tissue, held in place by a platinum frame (Fig. 1), was suspended with the anterior face of the brain just above or lightly resting on the chamber bottom. The enzyme-softened connective tissue sheath over the mushroom-body region was removed using a small-diameter glass pipette or fine forceps. Each brain was allowed to rest in continuously flowing oxygenated saline (95\% oxygen and $5 \%$ carbon dioxide) for at least 10 min before recording. Superfusion with oxygenated saline was continued throughout the recording period. Preparation was visualized using an upright microscope (Axioskop 2FS; Zeiss, Oberkochen, Germany) with a fixed stage and a $40 \times$ water immersion objective (Achroplan; numerical aperture, 0.8; Zeiss) and Nomarski optics. GFP was viewed with a BP 505-530 fluorescence filter. All record- ings were obtained from Kenyon cells located at the outer ring region of the mushroom bodies.

Electrophysiology. Current-clamp and voltage-clamp recordings were performed with whole-cell pipettes (10-14 M $\Omega$ ) filled with internal solution containing the following (in $\mathrm{mm}$ ): $102 \mathrm{~K}$-gluconate, $0.085 \mathrm{CaCl}_{2}$ $1.7 \mathrm{MgCl}_{2}, 17 \mathrm{NaCl}, 0.94$ EGTA, and 8.5 HEPES, pH 7.2, 235 Osm. All data shown were corrected for the $5 \mathrm{mV}$ liquid junction potential generated in these solutions. Recordings were made using a List EPC7 or an Axopatch 200B amplifier, a Digidata 1322A D-A converter (Molecular Devices, Foster City, CA), a Dell Dimension 8200 computer (Dell Computer, Round Rock, TX), and pClamp 9 software (Molecular Devices). Postsynaptic currents (PSCs) examined in the presence of TTX to block voltage-gated sodium channels were defined as miniature PSCs (mPSCs). The following drugs were bath applied in specific experiments: TTX (0.5 $\mu \mathrm{M})$, D-tubocurarine (curare; $20 \mu \mathrm{M}), \alpha$-BTX (5 $\mu \mathrm{M}), 6$-cyano7-nitroquinoxaline-2,3-dione (CNQX; $10 \mu \mathrm{M}), \mathrm{D}(-)$-2-amino-5phosphonopentanoic acid (APV; $100 \mu \mathrm{M}$ ), and picrotoxin (PTX; $10 \mu \mathrm{M}$ ).

In some cells, $0.4 \%$ biocytin was added to the internal pipette solution. After electrophysiological recording, the brain was fixed in phosphatebuffered $4 \%$ formaldehyde at $4^{\circ} \mathrm{C}$ for $1 \mathrm{~h}$. The brain was then incubated in an avidin-biotin peroxidase complex (ABC kit; Vector Laboratories, Burlingame, CA) for $90 \mathrm{~min}$, followed by $5 \mathrm{~min}$ at room temperature in 3',3-diaminobenzidine tetrahydrochloride. Brains were cleared in xylene, mounted in DPX (Sigma, St. Louis, MO), and examined on a light microscope. The neurons with bifurcated axons were classified as $\alpha / \beta$ lobe Kenyon cells, and those with nonbifurcated axons were classified as $\gamma$ lobe Kenyon cells. All recordings were done at room temperature, and only a single Kenyon cell was examined in each brain.

Analysis of synaptic currents. sPSCs and mPSCs were detected using mPSC detection software (MiniAnalysis; Synaptosoft, Decatur, GA) with a threshold criteria for individual events of 3 pA. In addition, events were accepted for kinetics analysis only if the shape was asymmetrical with a rising phase that peaked in $\leq 2.5 \mathrm{~ms}$ and a more slowly decaying falling phase. Current traces were filtered at $2 \mathrm{kHz}$ and digitized at $1-5 \mathrm{kHz}$ using pClamp 9.0 software. The mean amplitude and rise times were determined from the ensemble average currents assembled from $\geq 18$ single events in each neuron. Decay-time constants were determined by fitting a single exponential to the falling phase of the ensemble average current. Frequency was determined from a continuous recording of at least $3 \mathrm{~min}$ in duration.

\section{Results}

Kenyon cells are members of a spontaneously active neural circuit in the isolated adult whole-brain preparation

Entire brains, with the optic lobes and ommatidia attached, were removed from adult OK107 flies. A single brain was transferred to a glass-bottom chamber for electrophysiological recording. The mushroom-body regions were identified as two oval clusters of small (2-3 $\mu \mathrm{m}$ diameter), densely packed neurons located on either side of the midline, in the dorsal hemisphere on the posterior face of the brain (Fig. $2 \mathrm{~A}$ ). The majority of neurons visible on the surface in the mushroom-body region were Kenyon cells based on their expression of GFP (Fig. 2 A). In the present study, all of the recordings were from Kenyon cells located in the peripheral region of the mushroom bodies, where profiles of individual somata were clearly delineated (Fig. 2B). Whole-cell, current-clamp recordings from $10 \mathrm{GFP}+$ Kenyon cells revealed a mean resting potential of $-60.9 \pm 1.7 \mathrm{mV}$ (mean $\pm \mathrm{SEM}$ ). None of these Kenyon cells were firing spontaneous action potentials (APs), but 5 of 10 exhibited subthreshold spontaneous activity. A whole-cell, current-clamp recording from a GFP + Kenyon cell illustrates the activity pattern seen in four of five of the spontaneously active cells, with characteristic small-amplitude spontaneous depolarizations (Fig. 2C). A voltage-clamp recording from the same cell illustrates the underlying spontaneous inward currents (Fig. 2D). These data demonstrate that it is possible to record from identified Kenyon cells in the isolated adult fly brain, 

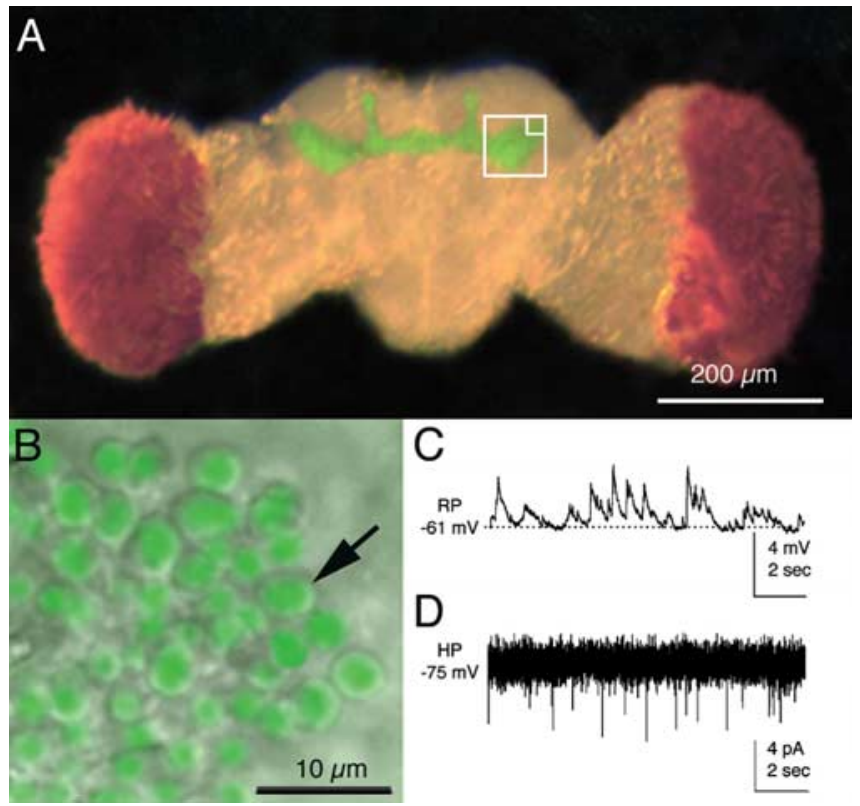

Figure 2. Spontaneous activity in Kenyon cells in an isolated whole-brain preparation. $\boldsymbol{A}$, GFP + Kenyon cell-body clusters are visible in the mushroom-body region (right hemisphere cluster delineated by large white square), on the posterior surface of an entire brain isolated from a 2-d-old 0K107 adult fly. Kenyon cell axons appear as L-shaped dorsomedial projections. A fluorescent mask projected on the Nomarski image. $\boldsymbol{B}$, Individual GFP + Kenyon cell bodies can be distinguished at higher magnification (top right region of square in $\boldsymbol{A}$ ) and targeted for electrophysiological recording in the living isolated brain preparation. C, Whole-cell, currentclamp recording of spontaneous depolarizations from a single Kenyon cell. RP, Resting potential. $\boldsymbol{D}$, Voltage-clamp recording of the underlying spontaneous currents from the same Kenyon cell. HP, Holding potential.

and the pattern of spontaneous activity suggests these neurons are members of an active synaptic network in this preparation.

To investigate the properties of the currents underlying the spontaneous activity, voltage-clamp recordings were obtained from GFP+ OK107 Kenyon cells $(n=109)$ in brains isolated from adult female flies between 1 and $5 \mathrm{~d}$ after eclosion (one neuron per brain). Whole-cell, voltage-clamp recordings, maintained on average of $22.7 \pm 1.7 \mathrm{~min}$ (range, 3-60 min), demonstrated the presence of spontaneous currents in 61\% (66 of 109) of the neurons.

Spontaneous activity patterns, monitored at a holding potential of $-75 \mathrm{mV}$, fell into three major groups. The most common group was characterized by the presence of discrete transient inward currents that had kinetic properties typical of sPSCs with a rapid rate of rise and slower decay phase (Fig. $3 A$ ). The sPSC frequency varied widely from cell to cell (range, $0.04-15.7 \mathrm{~Hz}$ ) with an average frequency of $2.7 \pm 0.53 \mathrm{~Hz}$ (mean \pm SEM). Approximately $74 \%$ (49 of 66) of the Kenyon cells were in this group, which was termed the sPSC class (Fig. 3D). The second activity pattern, observed in $12 \%$ of the Kenyon cells, was called the bursting sPSC class (Fig. $3 B, D$ ). This was defined by the appearance of clusters of sPSCs, in addition to the presence of discrete sPSCs between the bursts. The mean burst frequency in these cells was $0.23 \mathrm{~Hz}$. Single Kenyon cells were observed to switch from the sPSC to the bursting sPSC activity pattern during the course of the recording (4 of 49). Therefore, these activity states do not appear to define electrophysiologically distinct Kenyon cell types. Instead, they are likely to reflect differences in AP-dependent release of neurotransmitter in members of a synaptic network either directly or indirectly presynaptic to the Ke-

\section{A sPSCs}

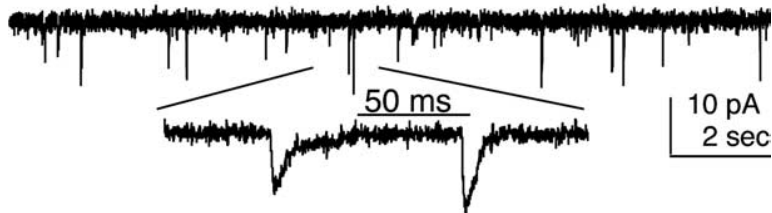

B Bursting sPSCs

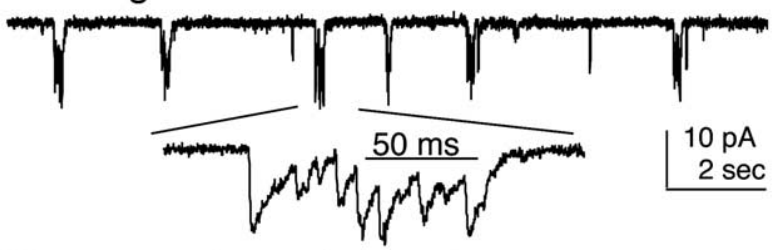

C Slow wave inward currents
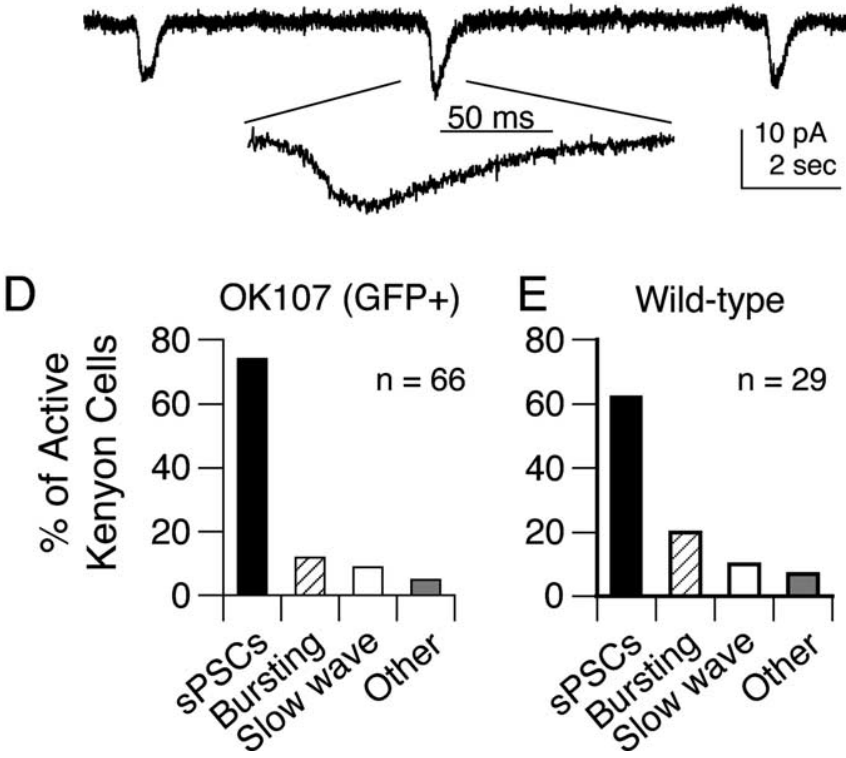

Figure 3. Spontaneous currents in Kenyon cells. A, Whole-cell, voltage-clamp recording from a GFP + OK107 Kenyon cell exhibiting the most common activity pattern (49 of 66 neurons) characterized by the presence of discrete sPSCS. Inset, High time resolution of the indicated region illustrates the rapid rise and slow decay phase of the SPSCs. Holding potential, -75 $\mathrm{mV}$. B, Activity pattern in a second GFP + OK107 Kenyon cell, classified as bursting sPSCs, was observed less often ( 8 of 66 ). This class was defined by clusters of fast synaptic currents. Inset, High time resolution of single burst. C, Slow-wave inward current activity, seen in 6 of 66 GFP + OK107 Kenyon cells, was dominated by large slowly rising and decaying inward currents. $\boldsymbol{D}$, Distribution of activity patterns, as a percentage of active GFP + Kenyon cells in 0K107. $\boldsymbol{E}$, Kenyon cells in wild-type brains were not significantly different ( $\chi^{2}$ analysis). The number of Kenyon cells with an activity pattern in each class was expressed as a percentage of the total number of active Kenyon cells in each genotype.

nyon cells. A third activity pattern, seen in $\sim 9 \%$ of the neurons, was dominated by large, slowly rising and decaying inward currents that occurred at a mean frequency of $0.21 \pm 0.05 \mathrm{~Hz}$ (Fig. $3 C, D)$. There were few or no events with classic synaptic current kinetics in these recordings. The mechanisms underlying generation of the slow-wave currents are unknown. Finally, there were a small number of Kenyon cells (2\%) in which activity was detected that could not be assigned to any of the three classes illustrated.

To determine whether GFP expression itself affects neuronal activity, recordings were also obtained from 51 Kenyon cells in Canton-S wild-type flies. The mushroom bodies were identified using Nomarski optics, and recordings were obtained from Kenyon cells located near the periphery of the mushroom bodies, 
the same region chosen in the GFP-expressing flies. In a subpopulation of neurons, inclusion of biocytin in the pipette and subsequent visualization of the stained cells at the conclusion of the recording confirmed their identity as $\alpha / \beta$ (7 of 11) or $\gamma$ ( 4 of 11) lobe Kenyon cells. In wild-type brains, $57 \%$ of the Kenyon cells were active, similar to the percentage of GFP+ Kenyon cells active in OK107 brains (61\%). All three classes of spontaneous activity were also observed in the wild-type Kenyon cells. Again, the sPSC class was the most common, including in the histologically identified $\alpha / \beta$ and $\gamma$ lobe Kenyon cells. The distribution between the three classes was not significantly different ( $\chi^{2}$ analysis) in wild-type and GFP $+\mathrm{Ke}$ nyon cells (Fig. $3 D, E$ ). Together, these data indicate that GFP expression does not alter the AP-dependent activity recorded in Kenyon cells.

nAChRs mediate most of the sodium AP-dependent excitatory drive in the absence of normal sensory input To further explore the role of AP-dependent release of neurotransmitter in contributing to the spontaneous currents observed in the Kenyon cells, synaptic current activity was monitored before and after bath application of TTX to block sodium channels. TTX resulted in a dramatic and significant reduction in the synaptic current frequency (Fig. $4 A, B$ ). The synaptic currents remaining in TTX were classified as mPSCs. Interestingly, no significant change in synaptic current amplitude was observed after application of TTX (Fig. 4C). Thus, although the majority of sPSCs in Kenyon cells require sodium AP-dependent release of neurotransmitter, these events appear to be mediated by release of a volume of neurotransmitter equivalent to that which gives rise to an mPSC. These data confirm that Kenyon cells are members of a spontaneously active synaptic network in the isolated brain preparation.

Studies in cell culture suggest that nAChRs are likely to be the predominant receptors mediating fast excitatory synaptic transmission in neurons throughout the adult Drosophila CNS ( $\mathrm{Su}$ and O'Dowd, 2003). As expected, the nAChR antagonist curare reversibly blocked the majority of the sodium AP-dependent synaptic activity in Kenyon cells (Fig. $4 D, E$ ). There was a significant reduction in mean sPSC frequency after the addition of curare or $\alpha$-BTX (Fig. $4 E$ ). These data show that $\alpha$-BTX-sensitive nAChRs represent a major receptor class regulating the spontaneous excitatory drive in the AP-dependent synaptic transmission recorded in Kenyon cells in situ.

\section{$\alpha$-BTX-sensitive nAChRs mediate mEPSCs in Kenyon cells}

To determine whether $\mathrm{nAChRs}$ also mediate fast transmission directly in Kenyon cells, pharmacological experiments were done in the presence of TTX to examine the AP-independent synaptic currents. The mPSC frequency was significantly reduced by bath application of curare or $\alpha$-BTX (Fig. 5A). These data demonstrate that the majority of the mPSCs in Kenyon cells in the isolated whole-brain preparation are mEPSCs mediated by nAChRs.

Because a small percentage of the Kenyon cells also exhibited nAChR-independent synaptic currents, which were not further characterized because of the low incidence and frequency, the following steps were taken to record cholinergic mEPSCs in isolation. All mEPSC recordings were done in the presence of GABA and glutamate receptor antagonists. In addition, after collection of data in this solution, the mEPSCs had to be blocked by the addition of a nAChR antagonist, curare or $\alpha$-BTX (Fig. 5B). In 2 of 16 Kenyon cells, there were
A Control (sPSCs)

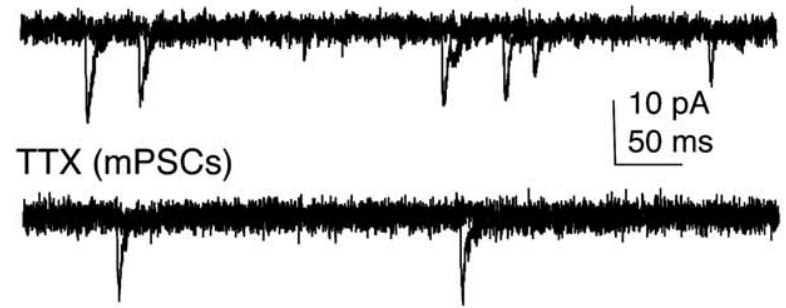

B
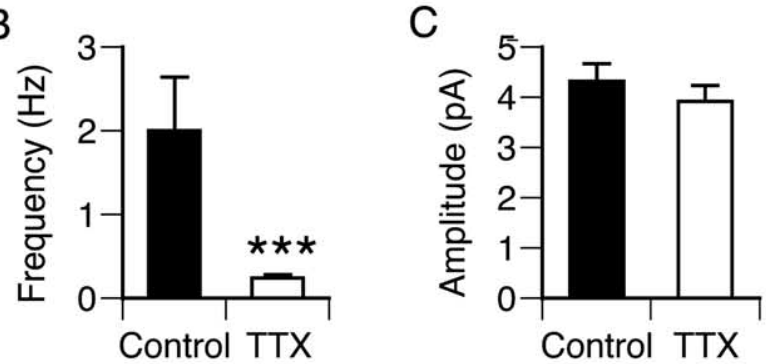

D

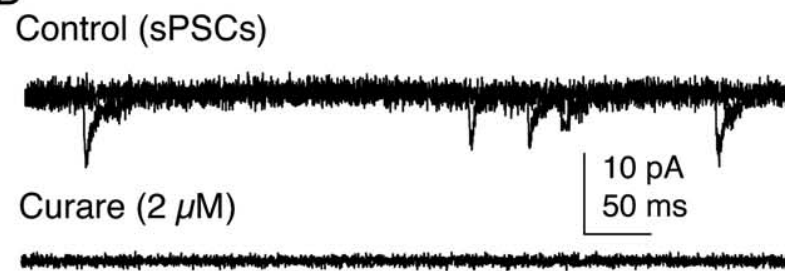

Wash

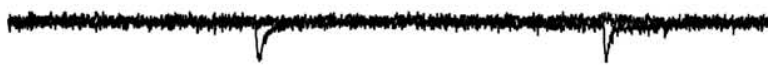

$E$

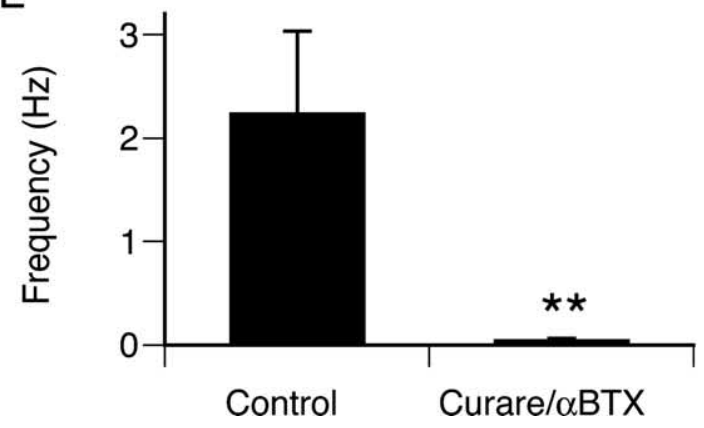

Figure 4. nAChRs mediate most of the spontaneous sodium AP-dependent excitatory drive in the Kenyon cell circuit in situ. $\boldsymbol{A}$, sPSCs recorded from a Kenyon cell in control saline. Bath application of TTX (1 $\mu \mathrm{m})$ blocked many of the synaptic currents. Events remaining in the presence of TTX were classified as MPSCs. Each record represents two superimposed current traces. $\boldsymbol{B}$, Bath perfusion of TTX caused a significant reduction in PSC frequency $\left({ }^{* * *} p<0.001\right.$, Wilcoxon signed rank test; $n=16$ ). $C$, Bath perfusion of TTX did not significantly reduce PSC amplitude. All recordings were made at a holding potential of $-75 \mathrm{mV}$. D, sPSCs recorded from a Kenyon cell in control saline were blocked by bath application of curare. Partial recovery of sPSCs after extensive washing is shown. Each record represents two superimposed current traces. $\boldsymbol{E}$, The sPSC frequency was significantly reduced by bath application of curare ( $20 \mu \mathrm{m}$; $n=6)$ or $\alpha \mathrm{BTX}(5 \mu \mathrm{m} ; n=4) .{ }^{* *} p<0.01$, Wilcoxon signed rank test $(n=10)$. Error bars indicate SEM.

a small number of events remaining after the addition of $\alpha$-BTX or curare, and data from these neurons were therefore excluded from further analysis. Analysis of the biophysical properties of cholinergic mEPSCs was based on data from the 
A

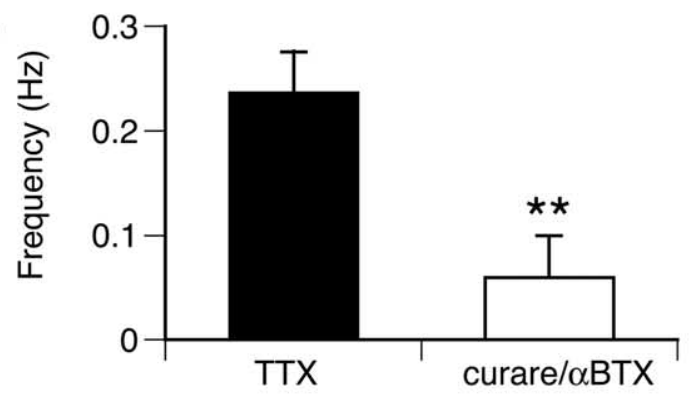

B

$T T X+P T X+A P V+C N Q X$

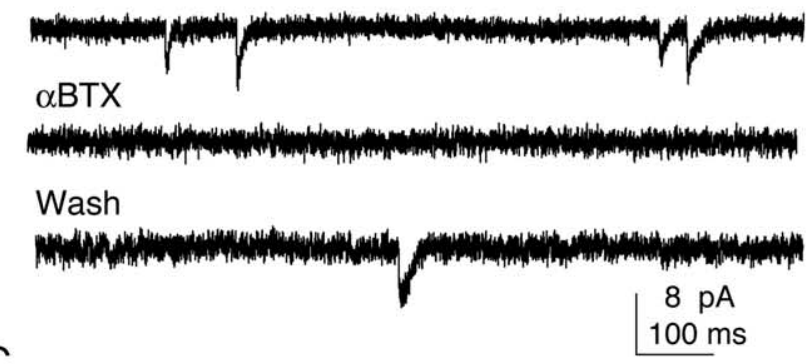

C
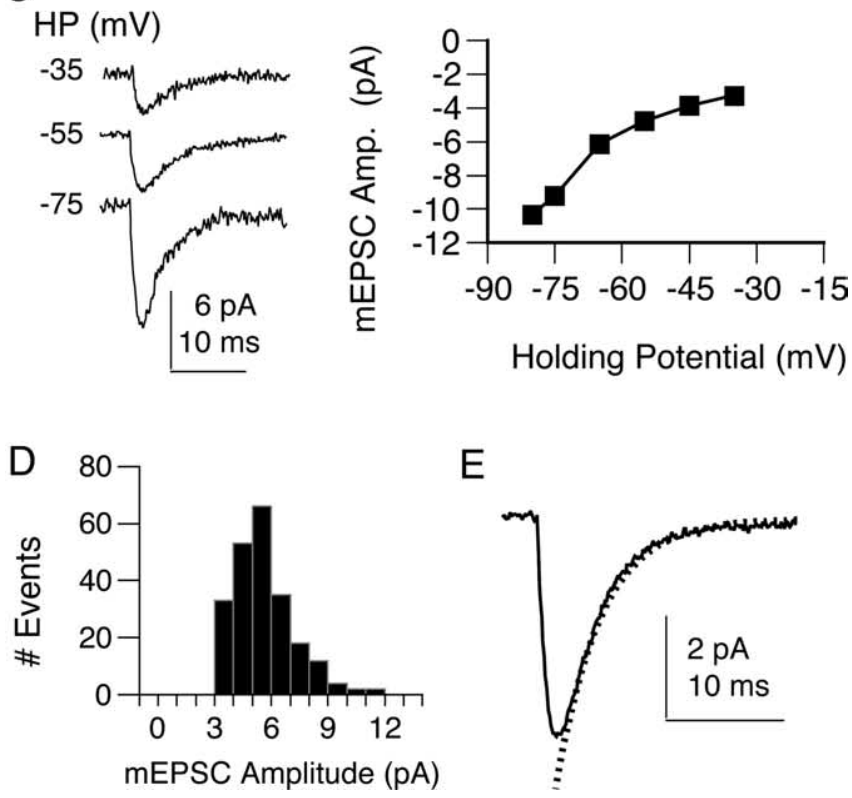

E

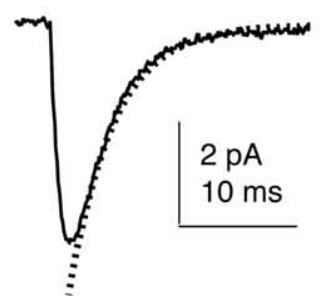

Figure 5. Sodium AP-independent synaptic currents mediated by $\alpha$-BTX-sensitive nAChRs in Kenyon cells in situ. $\boldsymbol{A}$, The frequency of mPSCs recorded in the presence of TTX was significantly reduced by the addition of curare $(20 \mu \mathrm{m} ; n=6)$ or $\alpha$-BTX $(5 \mu \mathrm{m} ; n=4)$. ${ }^{* *} p<0.01$, Wilcoxon signed rank test. $\boldsymbol{B}$, Sodium AP-independent synaptic currents recorded from a Kenyon cell in saline containing TTX, PTX, APV, and CNQX are completely blocked by bath perfusion of $5 \mu \mathrm{M} \alpha$-BTX, indicating they are mediated by nAChRs. Only partial recovery was observed after extensive washing. C, mEPSC amplitude decreases with increasing depolarization. Ensemble average mEPSCs constructed from 7-15 events at each holding voltage. HP, Holding potential. $\boldsymbol{D}$, The $\mathrm{mEPSC}$ amplitude histogram from a single cell reveals a typical, relatively narrow size distribution (mean, $4.5 \mathrm{pA}$ ). $\boldsymbol{E}$, The ensemble average $\mathrm{mEPSC}$ from the same cell, compiled from 225 individual events, exhibits a typical shape with a relatively fast rise and slow decay. The decay was fit with a single exponential (dotted line; $\tau=4 \mathrm{~ms}$ ). Error bars indicate SEM.

remaining 14 Kenyon cells in which all synaptic currents were sensitive to curare or $\alpha$-BTX.

Ensemble averages of the cholinergic mEPSCs in a single Kenyon cell generated at a series of holding voltages between -80 and $-35 \mathrm{mV}$ illustrates the typical current-voltage relationship
(Fig. 5C). The mEPSC amplitude decreased with increasing depolarization as we reported previously for cholinergic mEPSCs in cultured Drosophila Kenyon cells (Su and O'Dowd, 2003). However, reversal of the currents was not observed, presumably reflecting the location of the synaptic sites at regions electronically distant from the cell body. The properties of nAChRmediated mEPSCs were quantified in terms of frequency and biophysical properties. Cells were included in the analysis if at least 18 mEPSCs were recorded, and the criteria for inclusion of individual mEPSCs were a rise time $\leq 2.5 \mathrm{~ms}$ and an amplitude of $\geq 3 \mathrm{pA}$. The histogram generated from a single GFP+ Kenyon cell illustrates a relatively narrow mEPSC amplitude distribution, with event sizes ranging from 3 to $12 \mathrm{pA}$ and a mean of $4.5 \mathrm{pA}$ (Fig. $5 D$ ). The ensemble average mEPSC from the same cell reveals a relatively rapid rising phase and a slower decay phase (Fig. 5E).

Comparison of data from GFP + Kenyon cells in OK107 brains and from unlabeled Kenyon cells in wild-type brains did not reveal any striking differences in the frequency or biophysical properties of the cholinergic mEPSCs (Table 1). This indicates that GFP expression does not compromise electrophysiological analysis of these currents. A variety of studies indicate that Drosophila Kenyon cells, rather than being a homogenous group, consist of subpopulations that vary in both structure and function. To determine whether there were differences in the properties of the nAChRs mediating synaptic transmission in distinct subpopulations, it was important to be able to visualize these in the live brain, because each recording is quite labor intensive. This was not possible in OK107 brains because it is a pan-Kenyon cell driver line. Therefore, a different GAL4 line, 201Y, was used to drive GFP expression in a subpopulation of Kenyon cells, many of which project to the $\gamma$ lobe (Yang et al., 1995; Connolly et al., 1996). Cholinergic mEPSCs were recorded in both GFP+ and GFP - Kenyon cells in 201Y, and there were no significant differences between these two groups, nor between these groups and the data obtained from OK107 and wild type (Table 1). Although these data do not exclude subtle differences between cholinergic mEPSCs in the two groups, they show that $\alpha$-BTXsensitive nAChRs mediate mEPSCs in a broad spectrum of Kenyon cells, including $\gamma$ lobe neurons, in the isolated wholebrain preparation.

\section{Discussion}

Mushroom bodies play a central role in insect olfactory learning. Molecular genetic studies in Drosophila suggest that plasticity at synapses on mushroom-body Kenyon cell dendrites is involved in the processes of memory acquisition and storage. To test this hypothesis, however, it is necessary to record directly from Kenyon cells. Although local field potential recordings have been used to evaluate electrical activity in populations of neurons in the mushroom-body region (Nitz et al., 2002; van Swinderen and Greenspan, 2003), the only intracellular recordings of synaptic activity in Drosophila Kenyon cells had been from pupal neurons grown in dissociated cell culture (Su and O'Dowd, 2003). The preparation described in the present study allows assessment of AP-dependent and -independent synaptic activity in Kenyon cells within an isolated whole brain. This preparation is analogous, in a number of ways, to the "brain slice" that has been so important in evaluation of cellular mechanisms of synaptic transmission and plasticity in mammals. The brain is removed from the head of an adult fly, and whole-cell electrodes can be visually targeted to Kenyon cells in the mushroom-body region. Stable recordings from Kenyon cell bodies can be maintained for as 
long $1 \mathrm{~h}$. This preparation is equally useful for recording from neuronal groups throughout the brain including local and projection neurons in the antennal lobes (data not shown). One advantage of this preparation over the mammalian brain slice is that the entire adult fly brain fits in the recording chamber. Therefore, many of the neural circuits within the brain are likely to remain primarily intact, including those connecting the areas processing primary sensory information (antennal lobes) and association areas (mushroom bodies) (Heisenberg, 2003).

The GAL4 driver system was used to identify mushroom body Kenyon cells based on their expression of GFP. However, the cytoarchitecture of the mushroom-body region also allowed visual localization of Kenyon cells directly from the Nomarski image. Spontaneous activity and mEPSCs in Kenyon cells in wildtype and in OK107 flies were qualitatively and quantitatively similar. This confirms our conclusions from experiments in culture indicating that cytoplasmic GFP expression directed by the UAS-GFP transgene does not appear to alter neuronal function (Su and O'Dowd, 2003; Jiang et al., 2005). Thus, the UAS-GFP transgene in combination with different GAL4 driver lines is a useful marker for assessing the electrophysiological properties of synaptic transmission in neuronal populations that cannot be easily identified by their location. Use of the 201Y driver line, for example, allowed us to visually target our recording electrodes and assess activity in a subpopulation of Kenyon cells that had previously been shown to project primarily to the $\gamma$ lobe (Yang et al., 1995).

Blockade of the majority of the sodium AP-dependent and -independent spontaneous activity recorded in all Kenyon cells by curare and $\alpha$-BTX, indicates that nAChRs mediate most of the excitatory drive in cells that are directly and/or indirectly presynaptic to the Kenyon cells in the isolated brain. The direct input is likely to come from projection neurons in the antennal lobe based on anatomical studies indicating that these neurons are the primary source of cholinergic input to Kenyon cells in Drosophila (Bicker, 1999; Yasuyama et al., 2002). Furthermore, electrophysiological studies in the locust and honeybee demonstrate that cholinergic antennal lobe projection neurons provide direct excitatory afferent input to the mushroom bodies (Oleskevich, 1999; Perez-Orive et al., 2002). If the projection neurons mediate at least some of the spontaneous excitatory cholinergic input in Kenyon cells in the isolated brain, these neurons would have to be active in the absence of sensory input. Consistent with this hypothesis, a recent study has shown that antennal lobe projection neurons in the brain of intact adult Drosophila fire spontaneously even in the absence of applied odors (Wilson et al., 2004). In addition, recordings from projection neurons in our isolated brain preparation show that these cells fire spontaneous action potentials under the same recording conditions reported in the present study (data not shown). Future studies using VAChT mutants (Kitamoto et al., 2000; Kitamoto, 2002), or transgenes such as temperature-sensitive shibire in combination with the GAL4 driver line GH146 (Stocker et al., 1997), to modulate neurotransmitter release specifically in projection neurons will enable further assessment of the contribution of cholinergic currents to spontaneous excitatory inputs to Kenyon cells. Genetic manipulations to regulate activity in a cellspecific manner can also be used explore the role of antennal lobe neurons in modulating synaptic activity in Kenyon cells (Nitabach et al., 2002).

Although both Kenyon cells in situ and in vitro express fast synaptic currents mediated by nAChRs, it should be noted that the mEPSC amplitude was much smaller and the kinetics were slower in the entire brain compared with data obtained previously from cultured Kenyon cells [present study, our Fig. 5 and Table 1, vs Su and O'Dowd (2003), their Fig. 6]. In addition, the mEPSC amplitude in situ decreased with increasing depolarization at voltages between -80 and -35 , but the current size was too small to reliably measure at more depolarized potentials precluding the clear assessment of reversal potential. The most likely explanation for all of these observations is a greater electrotonic distance between the synaptic sites and the cell soma in situ versus dissociated culture. In cultured Kenyon cells, neurites from neighboring cells that are in close proximity contact the neurons both on the soma and neuritic processes. In situ, the majority of afferents to the Kenyon cell region form synapses on the dendrites that are located in the calyces, $>30 \mu \mathrm{m}$ from the cell-body region (Strausfeld et al., 2003), separated by a potentially passive length of membrane.

Functional studies in the locust and anatomical studies in the fly predict that Drosophila Kenyon cells, in addition to receiving excitatory cholinergic inputs, receive inhibitory input from GABAergic neurons (Perez-Orive et al., 2002; Yasuyama et al., 2002). In addition, Drosophila Kenyon cells grown in culture express PTX-sensitive GABA receptors that mediate inhibitory transmission. Consistent with the hypothesis that Kenyon cells in situ also express GABA receptors that mediate functional inhibition, there were AP-independent synaptic currents that remained after the addition of nAChR antagonists in some cells. Preliminary pharmacology indicated that some of these mPSCs were likely to be mediated by GABA receptors based on their blockade by PTX, but the low frequency of these events and the inability to reverse the blockade makes this identification tentative. In addition, there were some mPSCs detected in the combined presence of nAChR, GABA, and NMDA receptor antagonists. This suggests that fast synaptic transmission in Kenyon cells could also involve other receptor subtypes, such as dopamine and octopamine receptors, which are expressed at high levels in the mushroom bodies and influence aspects of olfactory associative learning (Han et al., 1996, 1998; Kim et al., 2003; Schwaerzel et al., 2003). Stimulation of specific populations while recording from Kenyon cells, in conjunction with pharmacology, will be critical in sorting out the contribution of the different receptors to fast synaptic transmission and plasticity in the Kenyon cells.

A variety of studies indicate that there are distinct subpopulations of Kenyon cells within the mushroom bodies that can be identified on the basis of their unique axonal projection patterns 
(i.e., $\alpha, \beta, \gamma$ ) and biochemical and developmental profiles (Yang et al., 1995; Crittenden et al., 1998; Lee et al., 1999; Strausfeld et al., 2003). Cell-specific blockade of synaptic transmission using the temperature-sensitive transgene shibire suggests that different Kenyon cell subpopulations may also have distinct functional roles. The $\gamma$ lobe Kenyon cells appear to be particularly important in learning and short-term memory (Zars et al., 2000), with retrieval of memory requiring signaling through the $\alpha / \beta$ lobes (McGuire et al., 2001). Our data indicate that the GFP+ neurons in the 201Y line, which labels predominantly $\gamma$ lobe Kenyon cells, have fast sodium AP-independent synaptic currents mediated by $\alpha$-BTX-sensitive nAChRs, similar to the general population of Kenyon cells that include $\alpha / \beta$ lobe neurons. However, these data do not allow us to rule out the possibility that there are differences in the specific nAChR subtypes in these two broad classes of Kenyon cells.

Because a large component of excitatory synaptic transmission, both AP dependent and independent, is mediated by $\alpha$-BTX-sensitive nAChRs in Drosophila Kenyon cells in situ, these receptors are likely to be involved in regulating cellular plasticity. Previous studies, using calcium-sensitive dyes, have documented that $\mathrm{nAChR}$ agonists, including acetylcholine and nicotine, induce rapid and reversible increases in intracellular calcium in Kenyon cells from the adult cricket (Cayre et al., 1999), honey bee (Bicker, 1996), and Drosophila (Yu et al., 2003; Jiang et al., 2005). Although our recent studies in cultured Drosophila Kenyon cells indicate that much of the calcium increase requires flux through voltage-gated calcium channels, some of the increase is attributable to calcium flux directly through the $\alpha$-BTX-sensitive nAChRs (J. M. Campusano, personal communication). Thus, synaptic activation of nAChRs in Kenyon cells via cholinergic afferents from the antennal lobes during repeated presentation of odorants could well lead to an increase in intracellular calcium sufficient to trigger changes in gene expression during associative learning. The ability to study synaptic transmission in a behaviorally relevant neural circuit now makes it possible to take full advantage of the genetic tools available in Drosophila to explore cellular links between gene expression and memory formation.

\section{References}

Baines RA (2003) Postsynaptic protein kinase A reduces neuronal excitability in response to increased synaptic excitation in the Drosophila CNS. J Neurosci 23:8664-8672.

Bicker G (1996) Transmitter-induced calcium signalling in cultured neurons of the insect brain. J Neurosci Methods 69:33-41.

Bicker G (1999) Histochemistry of classical neurotransmitters in antennal lobes and mushroom bodies of the honeybee. Microsc Res Tech 45:174-183.

Broadie KS, Rushton E, Skoulakis EMC, Davis RL (1997) Leonardo, a Drosophila 14-3-3 protein involved in learning, regulates presynaptic function. Neuron 19:391-402.

Cayre M, Buckingham SD, Yagodin S, Sattelle DB (1999) Cultured insect mushroom body neurons express functional receptors for acetylcholine, GABA, glutamate, octopamine, and dopamine. J Neurophysiol 81:1-14.

Connolly JB, Roberts IJH, Armstrong D, Kaiser K, Forte M, Tully T, O'Kane CJ (1996) Assoicative learning disrupted in impaired Gs signaling in Drosophila mushroom bodies. Science 274:2104-2107.

Crittenden JR, Skoulakis EM, Han KA, Kalderon D, Davis RL (1998) Tripartite mushroom body architecture revealed by antigenic markers. Learn Mem 5:38-51.

Davis RL (2004) Olfactory learning. Neuron 44:31-48.

Dubnau J, Grady L, Kitamoto T, Tully T (2001) Disruption of neurotransmission in Drosophila mushroom body blocks retrieval but not acquisition of memory. Nature 411:476-480.
Han K-A, Millar NS, Grotewiel MS, Davis RL (1996) DAMB, a novel dopamine receptor expessed specifically in Drosophila mushroom bodies. Neuron 16:1127-1135.

Han K-A, Millar NS, Davis RL (1998) A novel octopamine receptor with preferential expression in Drosophila mushroom bodies. J Neurosci 18:3650-3658.

Heisenberg M (2003) Mushroom body memoir: from maps to models. Nat Rev Neurosci 4:266-275.

Hodges DD, Lee D, Preston CF, Boswell K, Hall LM, O’Dowd DK (2002) tipE regulates $\mathrm{Na}^{+}$-dependent repetitive firing in Drosophila neurons. Mol Cell Neurosci 19:402-416.

Hou D, Suzuki K, Wolfgang WJ, Clay C, Forte M, Kidokoro Y (2003) Presynaptic impairment of synaptic transmission in Drosophila embryos lacking Gs $\alpha$. J Neurosci 23:5897-5905.

Jiang SA, Campusano JM, Su H, O’Dowd DK (2005) Drosophila mushroom body Kenyon cells generate spontaneous calcium transients mediated by PLTX-sensitive calcium channels. J Neurophysiol 94:491-500.

Kim YC, Lee HG, Seong CS, Han KA (2003) Expression of a D1 dopamine receptor dDA1/DmDOP1 in the central nervous system of Drosophila melanogaster. Gene Expr Patterns 3:237-245.

Kitamoto T (2002) Targeted expression of temperature-sensitive dynamin to study neural mechanisms of complex behavior in Drosophila. J Neurogenet 16:205-228.

Kitamoto T, Xie X, Wu CF, Salvaterra PM (2000) Isolation and characterization of mutants for the vesicular acetylcholine transporter gene in Drosophila melanogaster. J Neurobiol 42:161-171.

Lee D, O'Dowd DK (2000) cAMP-dependent plasticity at excitatory cholinergic synapses in Drosophila neurons: alterations in the memory mutant dunce. J Neurosci 20:2104-2111.

Lee T, Lee A, Luo L (1999) Development of the Drosophila mushroom bodies: sequential generation of three distinct types of neurons from a neuroblast. Development 126:4065-4076.

Marder E, Abbott LF, Turrigiano GG, Liu Z, Golowasch J (1996) Memory from the dynamics of intrinsic membrane currents. Proc Natl Acad Sci USA 93:13481-13486.

McGuire SE, Le PT, Davis RL (2001) The role of Drosophila mushroom body signaling in olfactory memory. Science 293:1330-1333.

Milner B, Squire LR, Kandel ER (1998) Cognitive neuroscience and the study of memory. Neuron 20:445-468.

Nitabach MN, Blau J, Holmes TC (2002) Electrical silencing of Drosophila pacemaker neurons stops the free-running circadian clock. Cell 109:485-495.

Nitz DA, van Swinderen B, Tononi G, Greenspan RJ (2002) Electrophysiological correlates of rest and activity in Drosophila melanogaster. Curr Biol 12:1934-1940.

Oleskevich S (1999) Cholinergic synaptic transmission in insect mushroom bodies in vitro. J Neurophysiol 82:1091-1096.

Park D, Coleman MJ, Hodge JJ, Budnik V, Griffith LC (2002) Regulation of neuronal excitability in Drosophila by constitutively active CaMKII. J Neurobiol 52:24-42.

Perez-Orive J, Mazor O, Turner GC, Cassenaer S, Wilson RI, Laurent G (2002) Oscillations and sparsening of odor representations in the mushroom body. Science 297:359-365.

Renger JJ, Ueda A, Atwood HL, Govind CK, Wu CF (2000) Role of cAMP cascade in synaptic stability and plasticity: ultrastructural and physiological analyses of individual synaptic boutons in Drosophila memory mutants. J Neurosci 20:3980-3992.

Rohrbough J, Broadie K (2002) Electrophysiological analysis of synaptic transmission in central neurons of Drosophila larvae. J Neurophysiol $88: 848$.

Rohrbough J, O’Dowd DK, Baines RA, Broadie K (2003) Cellular bases of behavioral plasticity: establishing and modifying synaptic circuits in the Drosophila genetic system. J Neurobiol 54:254-271.

Schwaerzel M, Monastirioti M, Scholz H, Friggi-Grelin F, Birman S, Heisenberg M (2003) Dopamine and octopamine differentiate between aversive and appetitive olfactory memories in Drosophila. J Neurosci 23:10495-10502.

Stocker RF, Heimbeck G, Gendre N, de Belle JS (1997) Neuroblast ablation in Drosophila P[GAL4] lines reveals origins of olfactory interneurons. J Neurobiol 32:443-456.

Strausfeld NJ, Sinakevitch I, Vilinsky I (2003) The mushroom bodies of Drosophila melanogaster: an immunocytological and golgi study of Ke- 
nyon cell organization in the calyces and lobes. Microsc Res Tech 62:151-169.

Su H, O'Dowd DK (2003) Fast synaptic currents in Drosophila mushroom body Kenyon cells are mediated by $\alpha$-bungarotoxin-sensitive nicotinic acetylcholine receptors and picrotoxin-sensitive GABA receptors. J Neurosci 23:9246-9253.

van Swinderen B, Greenspan RJ (2003) Salience modulates $20-30 \mathrm{~Hz}$ brain activity in Drosophila. Nat Neurosci 6:579-586.

Wilson RI, Turner GC, Laurent G (2004) Transformation of olfactory representations in the Drosophila antennal lobe. Science 303:366-370.

Yang MY, Armstrong JD, Vilinsky I, Strausfeld NJ, Kaiser K (1995) Subdivision of the Drosophila mushroom bodies by enhancer-trap expression patterns. Neuron 15:45-54.
Yao WD, Wu CF (2001) Distinct roles of CaMKII and PKA in regulation of firing patterns and $\mathrm{K}^{+}$currents in Drosophila neurons. J Neurophysiol 85:1384-1394.

Yasuyama K, Meinertzhagen IA, Schurmann FW (2002) Synaptic organization of the mushroom body calyx in Drosophila melanogaster. J Comp Neurol 445:211-226.

Yu D, Baird GS, Tsien RY, Davis RL (2003) Detection of calcium transients in Drosophila mushroom body neurons with camgaroo reporters. J Neurosci 23:64-72.

Zars T, Fischer M, Schulz R, Heisenberg M (2000) Localization of a shortterm memory in Drosophila. Science 288:672-675.

Zhong Y, Wu CF (1991) Altered synaptic plasticity in Drosophila memory mutants with a defective cyclic AMP cascade. Science 251:198-201. 